\title{
Different forms of cell death induced by putative BCL2 inhibitors
}

\author{
M Vogler ${ }^{1}, K_{\text {Keber }}{ }^{1}$, D Dinsdale ${ }^{1}$, I Schmitz ${ }^{2}$, K Schulze-Osthoff $^{3}$, MJS Dyer ${ }^{1}$ and GM Cohen ${ }^{\star, 1}$
}

Several inhibitors of BCL2 proteins have been identified that induce apoptosis in a variety of tumor cells, indicating their potential in cancer therapy. We investigated the specificity of six putative BCL2 inhibitors (obatoclax, gossypol, apogossypol, EM20-25, chelerythrine and ABT-737). Using cells deficient either for Bax/Bak or caspase-9, we found that only ABT-737 specifically targeted BCL2 proteins and induced apoptosis by activation of caspase-9, as only ABT-737 induced apoptosis was completely inhibited in cells deficient for Bax/Bak or caspase-9. Our data show that only ABT-737 is a specific BCL2 inhibitor and all other compounds investigated were not specific for BCL2 proteins. Furthermore, investigations of the effects of these compounds in primary chronic lymphocytic leukemic cells showed that all compounds induced certain biochemical hallmarks of apoptosis, such as release of cytochrome $c$ and caspase cleavage. However, they all caused strikingly different ultrastructural changes. ABT-737 induced all the characteristic ultrastructural changes of apoptosis together with early rupture of the outer mitochondrial membrane, whereas obatoclax, chlelerythrine and gossypol induced pronounced mitochondrial swelling with formation of phospholipid inclusions. Therefore, we conclude that biochemical measurements used earlier to define apoptosis like mitochondrial release of cytochrome $c$ and caspase cleavage, are insufficient to distinguish between classic apoptosis and other forms of cell death.

Cell Death and Differentiation (2009) 16, 1030-1039; doi:10.1038/cdd.2009.48; published online 24 April 2009

The evasion of cell death is one of the hallmarks of cancer and contributes to tumor progression, resistance to chemotherapy and treatment failure. ${ }^{1}$ Generally, cells can die primarily by apoptosis, a form of programmed cell death or after acute injury by cell and organelle swelling, disruption of cellular membranes and ultimately cell lysis/necrosis, which initiates an inflammatory response. ${ }^{2}$ In addition to these two major forms of cell death, cells may also die by autophagic cell death, cathepsin- or calpain-mediated cell death, anoikis, or caspase-independent apoptosis. ${ }^{3}$ Apoptosis can be triggered either at the cell surface (the extrinsic pathway) or at the mitochondria (the intrinsic pathway). In the intrinsic apoptotic pathway, cytochrome $c$ is released from the mitochondrial intermembrane space into the cytosol and induces apoptosome formation, with activation of caspase- 9 as the apical caspase.$^{4}$ Caspase- 9 activates the effector caspases-3 and -7 , which cleave several hundred cellular proteins, resulting in the characteristic biochemical and morphological features associated with apoptosis, including chromatin condensation, nuclear fragmentation and externalization of phosphatidylserine. ${ }^{5}$ In vivo, apoptotic cells maintain their plasma membrane integrity and are rapidly phagocytosed in the absence of an inflammatory response. ${ }^{2}$ However, in vitro at late times of incubation, apoptotic cells may undergo secondary necrosis, when the plasma membrane loses its integrity and increases its permeability. The release of cytochrome $c$ from mitochondria is mediated by the multidomain B-cell lymphoma 2 (BCL2) proteins, $\mathrm{BAX}$ and $\mathrm{BAK}$, which are regulated by antiapoptotic $\mathrm{BCL} 2$ proteins and $\mathrm{BH} 3-$ only proteins. Antiapoptotic $\mathrm{BCL} 2$ proteins comprise BCL2 itself, BCL-X $X_{L}, M C L 1, B C L w, B C L-B$, and BCL2A1 and contain a hydrophobic groove, which is formed by their BCL2 homology 1, 2 and 3 domains. Both BAX/ BAK and BH3-only proteins can bind into this hydrophobic groove and can thus be inhibited by antiapoptotic BCL2 proteins. ${ }^{6,7}$

Antiapoptotic BCL2 proteins are overexpressed in a variety of tumors and their expression often correlates with drug sensitivity. ${ }^{7,8}$ Owing to their important function in regulating cell death, pharmacological inhibition of BCL2 proteins is a promising strategy for apoptosis induction or sensitization to chemotherapy. The first approach targeting BCL2 used antisense nucleotides, and subsequently, several small molecule inhibitors have been developed. ${ }^{9,10}$ Most of these compounds have been identified by screening for binding to $\mathrm{BCL}-\mathrm{X}_{\mathrm{L}}$, and the resulting compounds are pan-BCL2 inhibitors that bind antiapoptotic BCL2 proteins with affinities ranging from subnanomolar to micromolar concentrations. A noteworthy exception is the development of ABT-737 and its orally active analog ABT-263, which was the result of NMR-based structural design. As a result, ABT-737 and

${ }^{1}$ MRC Toxicology Unit, Hodgkin Building, University of Leicester, Leicestershire, UK; ${ }^{2}$ Institute of Medical Microbiology and Hospital Hygiene, University of Duesseldorf, Duesseldorf, Germany and ${ }^{3}$ Interfaculty Institute of Biochemistry, University of Tuebingen, Tuebingen, Germany

${ }^{*}$ Corresponding author: GM Cohen, MRC Toxicology Unit, University of Leicester, Hodgkin Building, PO Box 138, Lancaster Road, Leicester, Leicestershire LE1 9HN, UK. Tel: + 44116 2525601; Fax: + 44116 2525616; E-mail: gmc2 @le.ac.uk

Keywords: Bcl2 inhibitors; mitochondria; ABT-737; obatoclax; chronic lymphocytic leukemia

Abbreviations: CLL, chronic lymphocytic leukaemia; DKO, double knock-out; FCS, fetal calf serum; MEFs, murine embryonic fibroblasts; MOMP, mitochondrial outer membrane permeabilization; MTT, 3-(4,5-dimethylthiazol-2-yl)-2,5-diphenyltetrazolium bromide; PARP, poly-(ADP-ribose) polymerase; PS, phosphatidylserine

Received 07.1.09; revised 11.3.09; accepted 23.3.09; Edited by S Kaufmann; published online 24.4.09 
ABT-263 bind selectively to BCL2, BCL- $X_{L}$ and $B C L w$ but have low affinities for MCL1, BCL-B and BCL2A1. ${ }^{11,12}$ ABT-737 induces apoptosis by binding to BCL2 and BCL- $X_{L}$ and releasing proapoptotic binding partners. ${ }^{13}$ In contrast, resistance to ABT-737 has been linked to high expression of MCL1. ${ }^{14,15}$ Other BCL2 antagonists also bind MCL1, and obatoclax (GX15-070) disrupts BAK binding to MCL1. ${ }^{16}$

In this study, we investigated the mechanism of cell death induction by gossypol, ${ }^{17}$ apogossypol, ${ }^{18}$ chelerythrine, ${ }^{19}$ obatoclax, ${ }^{16}$ EM20-25, ${ }^{20}$ and ABT-737. ${ }^{11}$ Gossypol, isolated from cotton seeds and used as a male contraceptive, was recently recognized as binding and interacting with antiapoptotic BCL2 family members and inducing apoptosis. ${ }^{17}$ Removal of the two reactive aldehyde groups in gossypol resulted in the synthesis of apogossypol, with similar binding kinetics to BCL2 and BCL- $X_{L}$ but with reduced toxicity in vivo. ${ }^{17,21}$ Initially described as a selective protein kinase $\mathrm{C}$ inhibitor, ${ }^{22}$ chelerythrine, a naturally occurring benzophenanthridine alkaloid, was subsequently identified by high throughput screening as an inhibitor of BCL-X ${ }_{\mathrm{L}}{ }^{19}$ EM20-25, which binds to the $\mathrm{BH} 3$ domain of $\mathrm{BCL} 2$, is a derivative of HA14-1, but lacks its effects on mitochondrial respiration. ${ }^{20}$ ABT-263, obatoclax and AT-101, the (-) enantiomer of gossypol, are in early clinical trials for lymphoid malignancies or solid tumors. ${ }^{23-25}$

However, despite the use of these inhibitors in preclinical mechanistic studies, proof for their specificity for BCL2 proteins is limited. Specific inhibitors of BCL2 proteins should induce apoptosis in a BAX/BAK-dependent manner with subsequent release of cytochrome $c$ and activation of caspase-9. Although it has been shown that several BCL2 inhibitors might activate the intrinsic apoptotic pathway, there is little evidence that activation of this pathway is required for cell death induction. Our studies now show that only ABT-737, of the six inhibitors studied, induces cell death by inhibition of BCL2 and activation of the intrinsic pathway with caspase-9 as the apical caspase. All the other inhibitors seem to induce cell death primarily by damaging mitochondria and have additional cellular targets that may result in significant nonmechanism based toxicities.

\section{Results}

Cell death induction in MEFs deficient in Bax and Bak. To test the specificity of BCL2 inhibitors, we initially used murine embryonic fibroblasts (MEFs), either wild type (wt) or deficient in both Bax and Bak (double knock out DKO). If cell death induction is specifically mediated by BCL2 proteins, Bax or Bak are required for release of cytochrome $c$ and cell death execution. Therefore in cells deficient in Bax and Bak, cell death arising from BCL2 inhibition should be completely inhibited. ${ }^{6,15}$ Amongst the BCL2 inhibitors tested, the most effective compound in MEFs was obatoclax, which was the only compound to induce cell death at submicromolar concentrations (Figure 1a). Using PS externalization as a measure of cell death, obatoclax was almost equally effective in killing both wt and DKO MEFs, showing that obatoclax is very effective in killing MEFs independently of Bax or Bak. To exclude any possible interference with this cell death assay owing to the strong inherent fluorescence of obatoclax, we also assessed the toxicity of obatoclax by two other methods, in which the assessment of toxicity was completely independent of any fluorescent signals. Firstly, viability was assessed using an MTT assay, which predominantly measures the ability of mitochondrial enzymes to reduce 3-4,5-dimethylthiazol2-yl)-2,5-diphenyltetrazolium bromide (MTT) to formazan. Obatoclax induced a similar concentration-dependent inhibition of viability or metabolic activity in both wt and DKO MEFs, with the wt MEFs being slightly more susceptible (Supplementary Figure 1a). Furthermore, obatoclax $(0.1 \mu \mathrm{M})$ inhibited colony formation of both wt and DKO MEFs to approximately similar extents (Supplementary Figure 1b). Taken together, these data show that obatoclax readily induces cell death in a Bax/Bak independent manner in MEFs.

Chelerythrine induced an almost identical concentrationdependent cell death in both wt and DKO MEFs (Figure 1b). Gossypol and apogossypol induced a concentration-dependent cell death in both wt and DKO MEFs but were clearly more potent in wt MEFs, indicating that Bax and/or Bak are involved in cell death induction (Figure 1c and d). However, at higher concentrations of apogossypol $(30 \mu \mathrm{M})$, cell death was also induced in DKO MEFs, suggesting that at high concentrations, these compounds have other targets in addition to BCL2 family proteins. EM20-25 was a weak inducer of cell death in wt MEFs (Figure 1e). It is to be noted that, ABT-737 induced cell death in wt but not in DKO MEFs, even at much higher concentrations of $\mathrm{ABT}-737(30 \mu \mathrm{M})$ (Figure 1f). These data show that only ABT-737, amongst the BCL2 inhibitors tested, is a specific inhibitor of BCL2. Cell death induced by obatoclax and chelerythrine seemed to be completely independent of BCL2 proteins, whereas cell death induction by gossypol and apogossypol seemed to be only partially due to their BCL2 inhibitory function.

\section{Cell death induction in cells deficient in caspase-9.}

Next, we investigated whether cell death induced by BCL2 inhibitors occurs through activation of the intrinsic apoptotic pathway. To extend our studies from a nonmalignant murine cell line to human leukemic cells, we used Jurkat T-cells deficient in caspase- 9 and the same cells reconstituted with caspase- 9 , as a control. ${ }^{26,27}$ Caspase- 9 is the initiator caspase in the intrinsic pathway and if apoptosis occurs through this pathway, it cannot proceed in the absence of caspase-9. Cell death was assessed by PS externalization for all the compounds except obatoclax. To exclude possible interference of the fluorescence of obatoclax, apoptosis was assessed by changes in the scatter properties of the cells (FSC/SSC). Both obatoclax (Figure 2a) and apogossypol (Figure 2d) induced cell death irrespective of caspase-9 expression, indicating that cell death did not occur as a consequence of activating the intrinsic apoptotic pathway. Upon exposure to chelerythrine (Figure 2b) or gossypol (Figure 2c), cell death was only slightly less in caspase-9 deficient cells as compared with caspase- 9 expressing cells, indicating that both chelerythrine and gossypol also induced cell death mainly independently of caspase- 9 and not primarily through the intrinsic apoptotic pathway. EM20-25 $(1-30 \mu \mathrm{M})$ did not induce cell death in Jurkat cells 

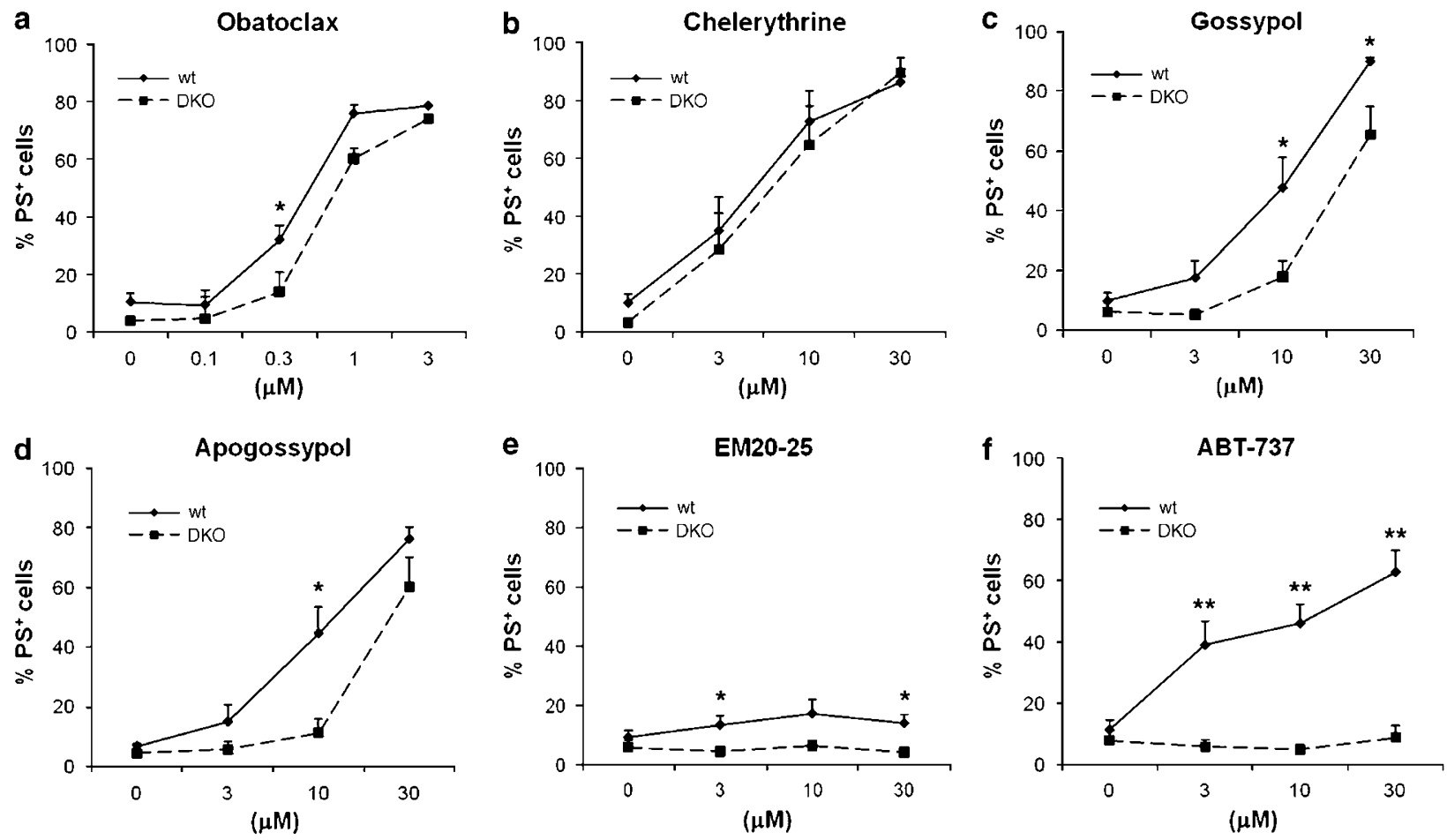

Figure 1 Cell death induced by putative BCL2 inhibitors is mainly independent of Bax and Bak. Wild type (wt) MEFs (solid lines) or Bax/Bak double knockout (DKO) MEFs (dotted lines) were exposed to the indicated concentrations of obatoclax (a), chelerythrine (b), gossypol (c), apogossypol (d), EM20-25 (e), or ABT-737 (f) for $48 \mathrm{~h}$. In (a and b) cell death was assessed by phosphatidylserine (PS) externalization and binding of Annexin-APC. In (c-f) cell death was assessed by PS externalization, binding of AnnexinFITC and PI staining. Data are mean + S.E.M. of $5-8$ experiments $\left({ }^{\star} P<0.05,{ }^{\star \star} P<0.01\right)$

(Figure 2e). ABT-737 induced a concentration-dependent cell death in cells expressing caspase-9 (Figure 2f). In cells deficient for caspase-9, ABT-737 induced cell death was completely inhibited, showing that ABT-737 induces cell death selectively through the intrinsic apoptotic pathway, which is absolutely dependent on caspase-9. Taken together, the experiments in Figure 1 and 2 show that all of the putative BCL2 inhibitors tested, with the exception of ABT-737, induce cell death mainly in a non-specific way that requires neither Bax/Bak nor caspase-9.

ABT-737 is $>100$-fold more potent at inducing death of CLL cells than other BCL2 inhibitors. Irrespective of their additional cellular targets and their mode of killing, the BCL2 inhibitors investigated in this study, several of which are already in clinical trials, might be valuable for cancer therapy. To investigate which compound was most efficient in killing primary leukemia cells, we exposed freshly isolated chronic lymphocytic leukemia (CLL) cells from patients to the different BCL2 inhibitors. We have shown earlier that freshly isolated CLL cells express both BAX and BAK and BCL2 and MCL1 but do not express detectable levels of $\mathrm{BCL}-\mathrm{X}_{\mathrm{L}}{ }^{28}$ Only $\mathrm{ABT}-737$ and chelerythrine induced PS externalization of CLL cells after $4 \mathrm{~h}$ exposure (Figure $3 \mathrm{a}$ ), whereas all the compounds induced PS externalization after $24 \mathrm{~h}$ (Figure 3b). ABT-737 induced cell death at low nanomolar concentrations after only $4 \mathrm{~h}$ of exposure (Figure 3a), whereas micromolar concentrations of chelerythrine were required to induce such rapid cell death Obatoclax, gossypol, apogossypol and EM20-25 all required micromolar concentrations and longer incubation times for cell death induction (Figure $3 b$ ). For comparison, at $24 \mathrm{~h}$ the $\mathrm{EC}_{50}$ of $\mathrm{ABT}-737$ was $9.4 \mathrm{nM}$, whereas the $\mathrm{EC}_{50}$ of obatoclax was $\sim 2 \mu \mathrm{M}$ and of chelerythrine $\sim 1.1 \mu \mathrm{M}$. Owing to the concentrations used, it was not possible to calculate an $\mathrm{EC}_{50}$ for gossypol, apogossypol and EM20-25. In conclusion, we found that ABT-737 was $>100$-fold more potent at inducing apoptosis in CLL cells than any of the other putative BCL2 inhibitors (Figure 3 ).

Next, we investigated which form of cell death was induced by the BCL2 inhibitors in CLL cells. Owing to the higher potency of ABT-737 compared with the other BCL2 inhibitors, these experiments were carried out with $10 \mathrm{nM}$ ABT-737 and $10 \mu \mathrm{M}$ of the other BCL2 inhibitors. The release of cytochrome $c$ from the mitochondrial intermembrane space to the cytosol after mitochondrial outer membrane permeabilization (MOMP) is considered to be an essential component of the intrinsic apoptotic pathway ${ }^{4}$ Release of cytochrome $c$ from mitochondria to cytosol was induced by all BCL2 inhibitors tested (Figure 4a). It is to be noted that, chelerythrine induced a very rapid and extensive cytochrome $c$ release within $30 \mathrm{~min}$ of exposure, whereas ABT-737 induced cytochrome $c$ release within $2 \mathrm{~h}$ of exposure. In line with their kinetics of apoptosis induction, obatoclax, gossypol, apogossypol and EM20-25 required longer incubation times but release of cytochrome $c$ was evident after $8 \mathrm{~h}$ of exposure. 

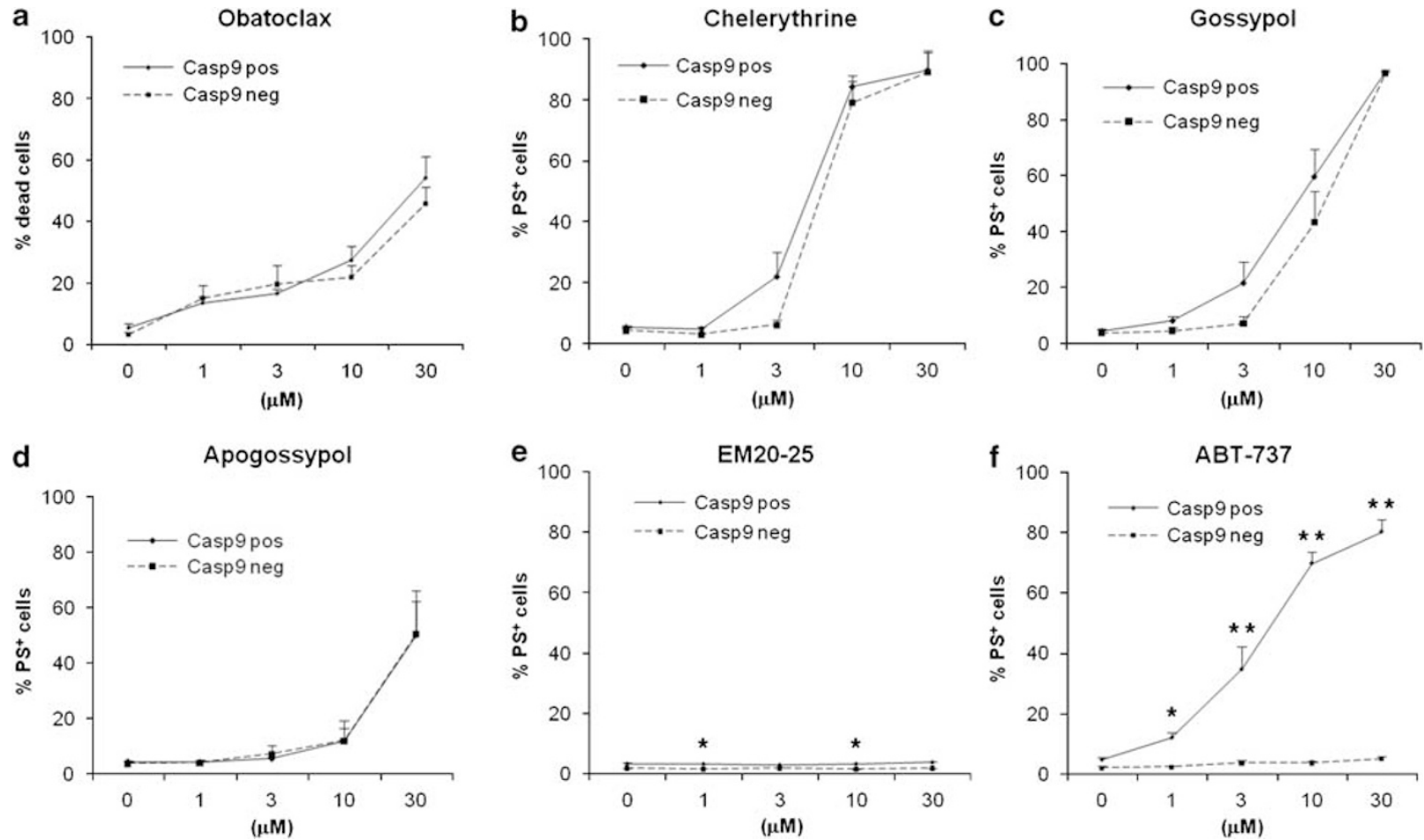

Figure 2 Cell death induced by putative BCL2 inhibitors is independent of caspase-9. Jurkat T-cells deficient in caspase-9 (dotted lines) or with reconstituted caspase-9 (solid lines) were exposed to the indicated concentrations of obatoclax (a), chelerythrine (b), gossypol (c), apogossypol (d), EM20-25 (e), or ABT-737 (f) for $24 \mathrm{~h}$. In (a) cell death was assessed by FSC/SSC analysis, in (b) cell death was assessed by phosphatidylserine (PS)-exposure and binding of Annexin-APC, and in (c-f) cell death was assessed by PS-exposure, binding of Annexin-FITC and PI staining. Data are mean + S.E.M. of 4 experiments $\left({ }^{\star} P<0.05,{ }^{\star \star} P<0.01\right)$
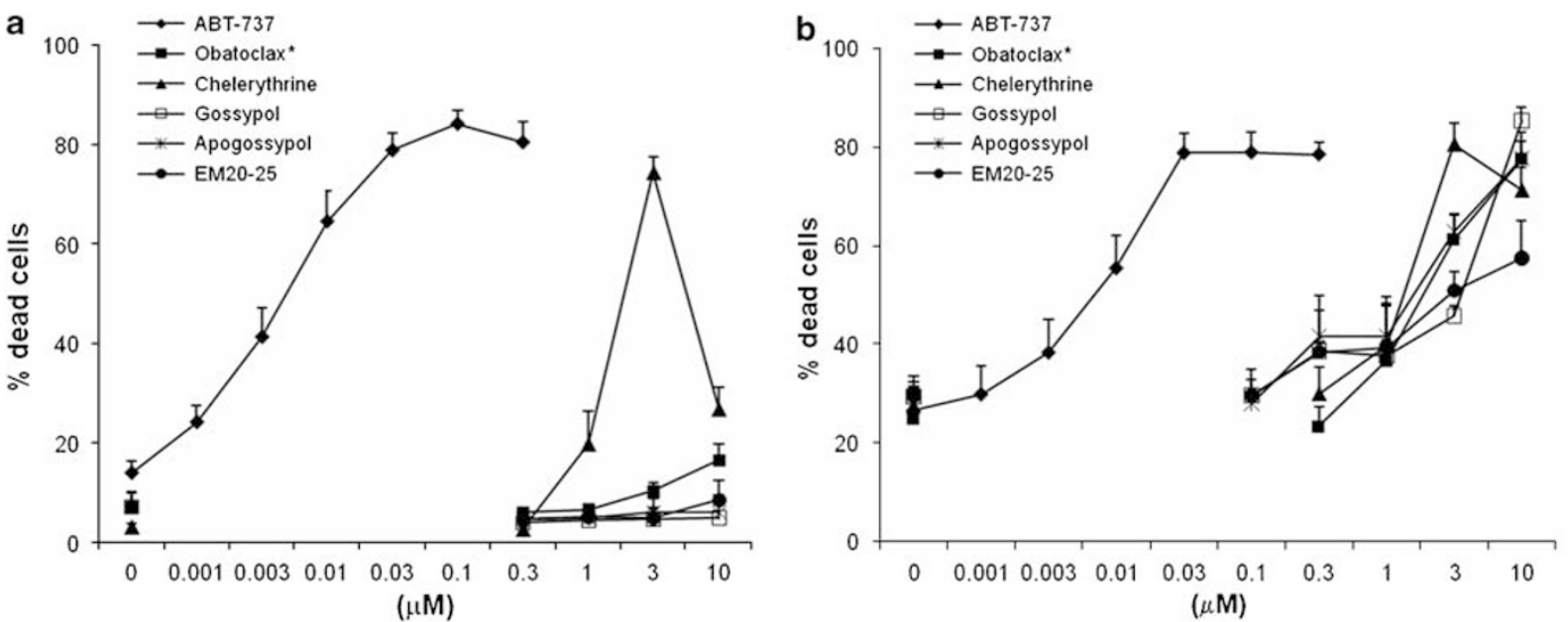

Figure 3 ABT-737 is most effective at killing primary CLL cells. CLL cells freshly isolated from the peripheral blood were exposed to the indicated concentrations of $B C L 2$ inhibitors for $4 \mathrm{~h}$ (a) or $24 \mathrm{~h}$ (b). For ABT-737, gossypol, apogossypol and EM20-25, cell death was assessed by phosphatidylserine (PS)-exposure, binding of Annexin-FITC and PI staining. For chelerythrine, cell death was assessed by PS externalization and binding of Annexin-APC, and for obatoclax cell death was assessed by FSC/SSC analysis. Data are mean + S.E.M. of cells from 15 individual patients

Besides release of cytochrome $c$, the cleavage and activation of caspases is a hallmark of apoptosis. To this end, we analyzed cleavage of caspases after exposure of CLL cells to BCL2 inhibitors. All BCL2 inhibitors were able to induce a certain extent of caspase cleavage. Chelerythrine induced a rapid cleavage of caspase- 3 and its canonical substrate poly-(ADP-ribose) polymerase (PARP), whereas caspase-9 cleavage was minor. ABT-737 induced cleavage of caspase- 3 , caspase- 9 and PARP within $2 \mathrm{~h}$ of exposure, whereas caspases and PARP were cleaved by obatoclax, gossypol, apogossypol and EM20-25 only at later time points. In conclusion, these data show that in CLL cells, all BCL2 


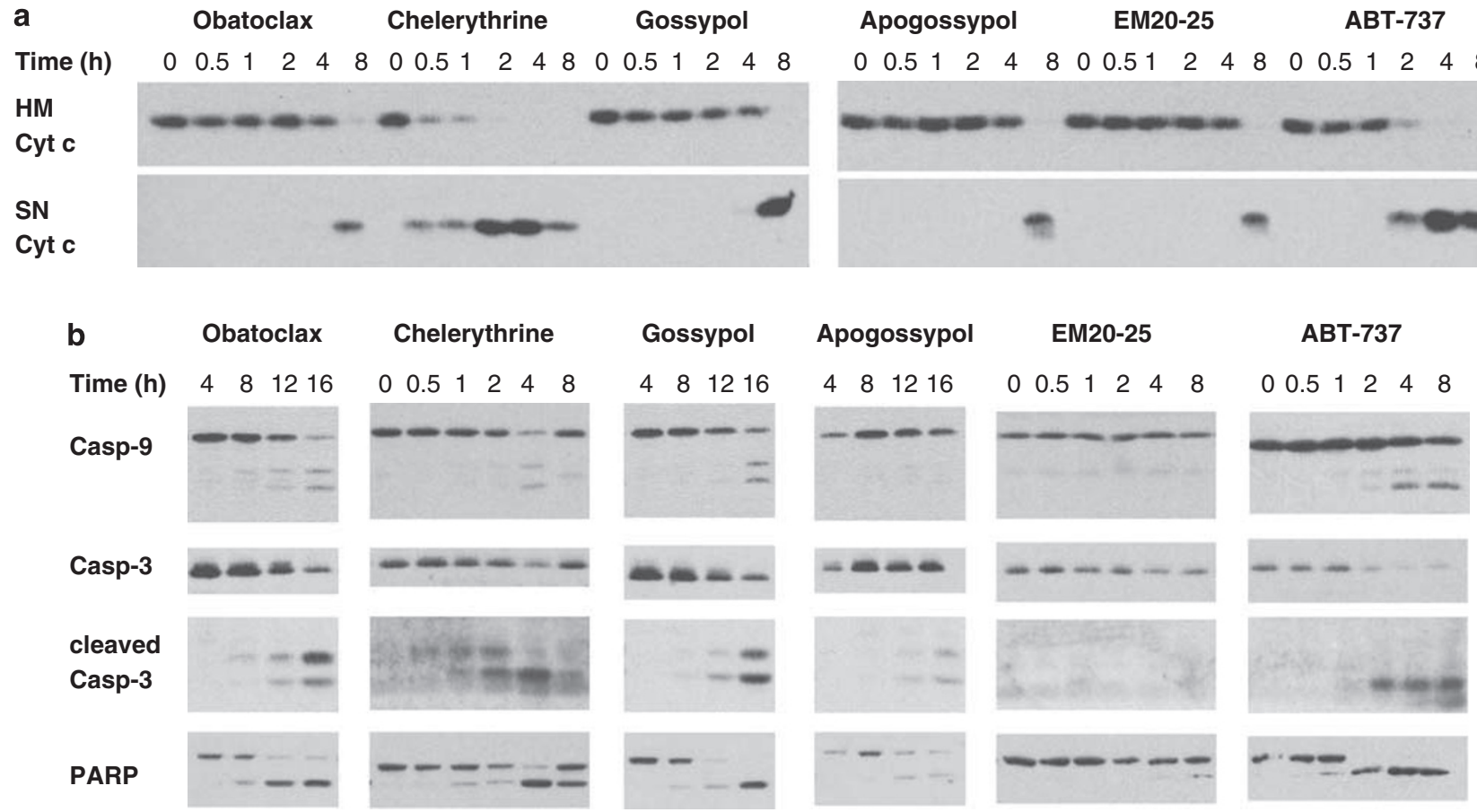

Figure $4 \mathrm{BCL} 2$ inhibitors induce cytochrome $c$ release and caspase cleavage. CLL cells freshly isolated from peripheral blood were exposed to $10 \mu \mathrm{M}$ obatoclax, $10 \mu \mathrm{M}$ chelerythrine, $10 \mu \mathrm{M}$ gossypol, $10 \mu \mathrm{M}$ apogossypol, $10 \mu \mathrm{M}$ EM20-25 or $10 \mathrm{nM}$ ABT-737 for the indicated time. (a) Release of cytochrome $c$ from mitochondria into cytosol was assessed by fractional lysis with $0.05 \%$ digitonin and isolation of heavy membranes (HM) containing mitochondria and supernatant (SN)-containing cytosol. (b) Cleavage of caspases and the caspase substrate, PARP, was assessed by western blotting. Results shown are from one experiment representative of three

inhibitors tested induce classic biochemical hallmarks of apoptosis, such as mitochondrial cytochrome $c$ release and caspase cleavage, albeit with very different kinetics and concentrations.

BCL2 inhibitors induce mitochondrial damage. In addition to biochemical analyses, we investigated the ultrastructure of CLL cells both untreated (Figure 5a) and upon exposure to BCL2 antagonists. Strikingly diverse ultrastructures were observed in CLL cells exposed to the putative BCL2 inhibitors with mitochondrial alterations being most prevalent (Figure 5). Obatoclax $(10 \mu \mathrm{M})$ induced massive mitochondrial swelling and loss of cristae within $1 \mathrm{~h}$ of exposure but both inner and outer mitochondrial membranes remained intact and no detectable alterations in nuclear structure were observed (Figure 5b). Many mitochondria contained multilaminated phospholipid whorls, which probably originated from cristae membranes. At later times ( $8 \mathrm{~h})$, some cells showed classical apoptotic nuclei with condensed chromatin (Figure 5c). Similarly, exposure of CLL cells to chelerythrine for only $1 \mathrm{~h}$ resulted in massively swollen mitochondria and inclusions showing concentric layers of phospholipid (Figure $5 \mathrm{~d}$ ). The mitochondrial changes were even more marked at $4 \mathrm{~h}$ (Figure $5 \mathrm{e}$ ), when they were accompanied by outer mitochondrial membrane discontinuities and striking nuclear changes. The nuclear changes, which were particularly evident after exposure to lower concentrations ( $3 \mu \mathrm{M})$ of chelerythrine (Figure $5 \mathrm{f}$ ), were characterized by the partial condensation of chromatin, similar to those we have described earlier involving the cleavage of chromatin into large kilobase pair fragments of 50 and $250 \mathrm{kbp}$ in the absence of internucleosomal cleavage. ${ }^{29}$ Signs of secondary necrosis, rare at $4 \mathrm{~h}$, were common after exposure to chelerythrine for $8 \mathrm{~h}$ (data not shown). Gossypol required longer incubation times before ultrastructural changes were detected. After exposure to gossypol for $12 \mathrm{~h}$, although most nuclei were unaffected, some apoptotic nuclei were clearly observed (Figure 6a). Gossypol also induced some mitochondrial swelling and loss of cristae accompanied by phospholipid inclusions, although this was much less common than with obatoclax and chelerythrine (Figure 6b). At this and later times, there was an increase in cells undergoing apoptosis and secondary necrosis. Taken together, these putative BCL2 antagonists all induced severe mitochondrial damage before cell death induction. Exposure to apogossypol for $12 \mathrm{~h}$ induced no significant mitochondrial swelling but interestingly caused a proliferation of the endoplasmic reticulum in localized clusters (Figure $6 \mathrm{c}$ and $\mathrm{d}$ ). However some cells with pyknotic nuclei, which were often undergoing secondary necrosis, were also observed (Figure 6e). In line with our earlier data, ${ }^{30} \mathrm{ABT}-737$ induced mitochondrial swelling and discontinuities in the outer mitochondrial membrane in addition to the characteristic ultrastructural changes of lymphocyte apoptosis (Figure 6f). No mitochondrial phospholipid inclusions were observed after exposure to ABT-737. Interestingly, although four compounds in this study induced swelling of mitochondria, only ABT-737 induced significant rupture of the outer mitochondrial membrane, as one of the earliest detectable lesions. 

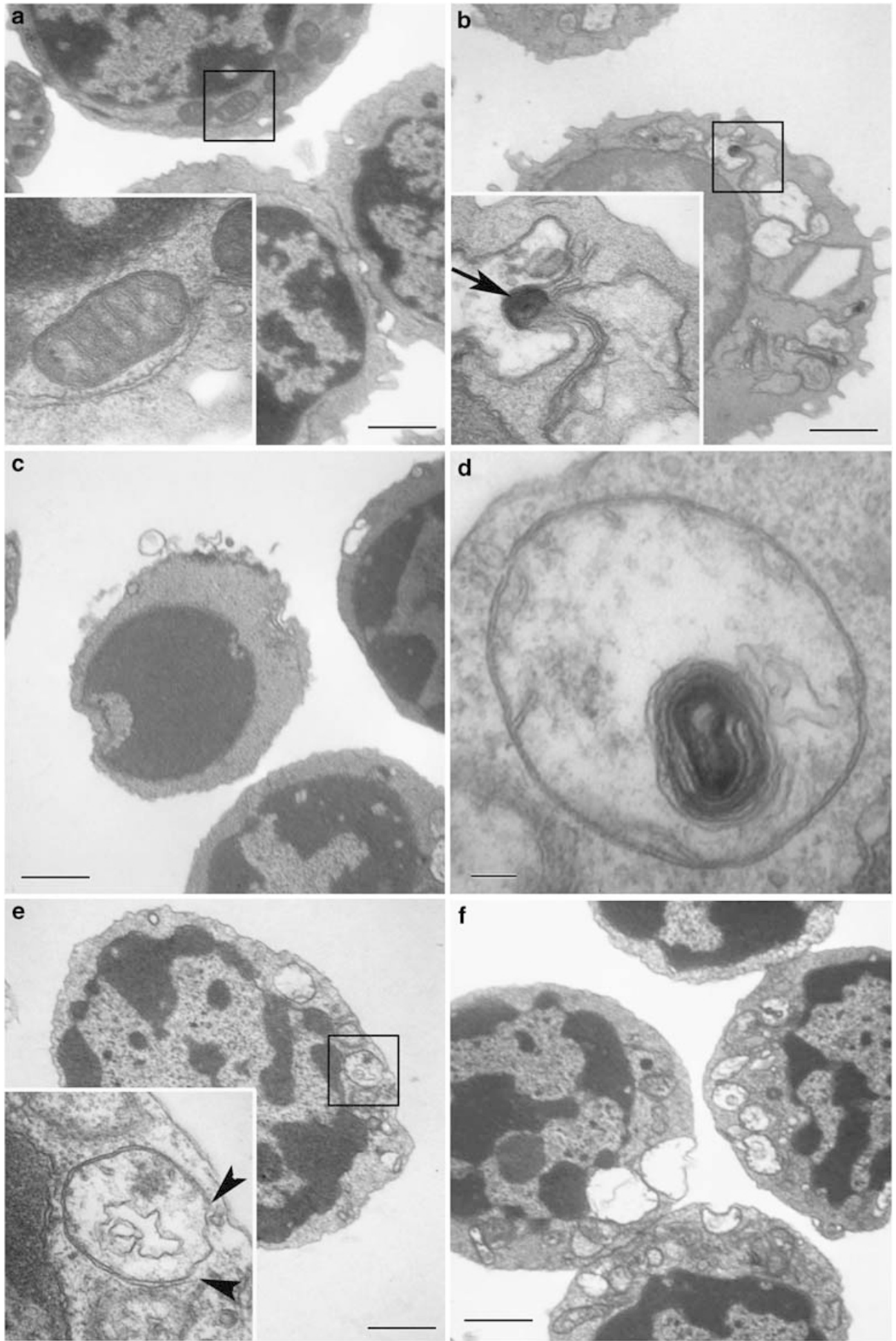

Figure 5 Obatoclax and chelerythrine induce mitochondrial damage. (a) Untreated CLL cells have mitochondria with well-defined transverse cristae. (b) Exposure to obatoclax $(10 \mu \mathrm{M})$ induced massive mitochondrial swelling and loss of cristae after $1 \mathrm{~h}$ of exposure. The inner and outer mitochondrial membranes remained intact and no detectable alterations in nuclear structure were observed. A multilaminated phospholipid whorl (black arrow) is evident in one of the swollen mitochondria. (c) Exposure to obatoclax $(10 \mu \mathrm{M})$ for longer times $(8 \mathrm{~h})$ also resulted in several cells exhibiting chromatin condensation characteristic of apoptosis. (d) Mitochondrial swelling, with loss of cristae but maintenance of mitochondrial membranes, occurred within $1 \mathrm{~h}$ of exposure of CLL cells to chelerythrine $(10 \mu \mathrm{M})$. These changes were sometimes associated with the development of whorls of phospholipid within the mitochondrial matrix. (Bar $=100 \mathrm{~nm})$. (e) After $4 \mathrm{~h}$ of exposure to chelerythrine $(10 \mu \mathrm{M})$, mitochondrial changes were accompanied by outer mitochondrial membrane discontinuities (black arrowheads) together with partial chromatin condensation in the nucleus. (f) Exposure to lower concentrations of chelerythrine $(3 \mu \mathrm{M})$ also induced mitochondrial and nuclear changes. In this Figure the scale bar $=1 \mu \mathrm{m}$, unless stated otherwise 

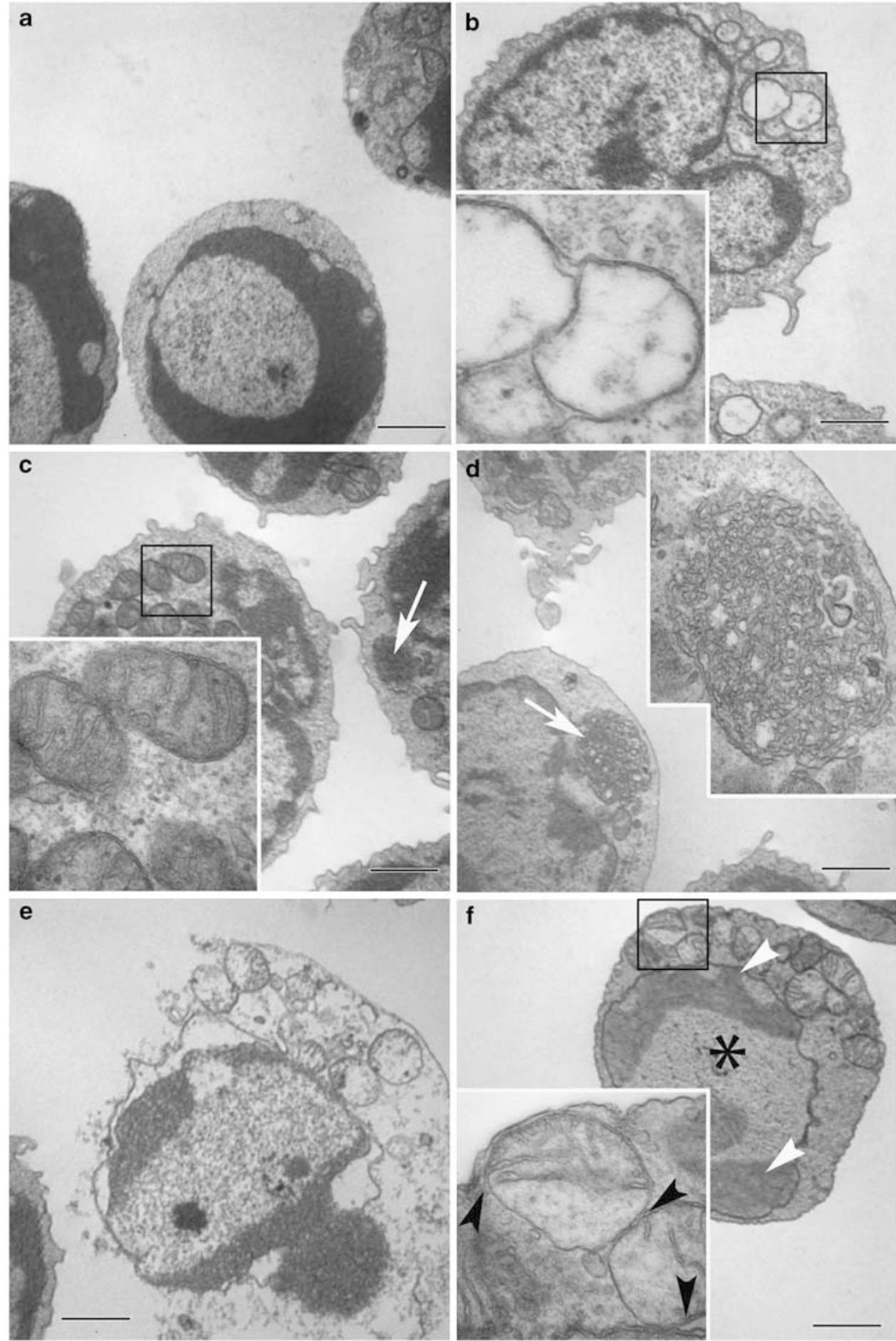

Figure 6 Different ultrastructural changes induced by gossypol, apogossypol and ABT-737. (a and b) Exposure to gossypol (10 $\mu \mathrm{M})$, for $12 \mathrm{~h}$, resulted in both the induction of characteristic apoptotic nuclei (a) and mitochondrial swelling and loss of cristae (b) in a small proportion of cells. (c) Mitochondria in cells exposed to apogossypol (10 $\mu \mathrm{M}$ ), for $12 \mathrm{~h}$, were indistinguishable from controls but localized clusters of endoplasmic reticulum (white arrow) were observed in many of these cells. (d) A higher magnification shows the proliferation of the endoplasmic reticulum in the apogossypol $(10 \mu \mathrm{M})$ exposed cells. (e) In addition apogossypol (10 $\mu \mathrm{M})$ resulted in some cells with pyknotic nuclei, which were often undergoing secondary necrosis. (f) Exposure to ABT-737 (10 nM) induced mitochondrial swelling and discontinuities in the outer mitochondrial membrane (black arrowheads) within $2 \mathrm{~h}$. Apoptotic nuclear changes including chromatin condensation (white arrowheads) and nucleolar disintegration (asterisk) were also common but no phospholipid inclusions were found in any mitochondria. In this Figure, the scale bar $=1 \mu \mathrm{m}$

Breakdown of this membrane after chelerythrine treatment at $4 \mathrm{~h}$ and much later after the other agents probably results from the rapid onset of secondary necrosis. In conclusion, all the putative BCL2 antagonists induce strikingly different morphological changes, implying significant differences in the biochemical pathways by which they induce cell death. 


\section{Discussion}

In this study, we investigated the specificity of BCL2 antagonists and their mechanisms of cell death induction. Although $A B T-737$ selectively binds $B C L 2, B C L-X_{L}$ and $B C L w$ but does not bind MCL1, BCL2A1 or BCL-B, ${ }^{11}$ all other inhibitors investigated here bind to all antiapoptotic BCL2 proteins with comparable affinities. ${ }^{9,10}$ It is to be noted that, ABT-737 binds BCL2 and BCL- $X_{L}$ with higher affinity than any of the other BCL2 inhibitors. The specificity of BCL2 inhibitors for BCL2 proteins can be investigated using cells that are deficient in Bax and Bak. Our data (Figure 1) strongly suggest that, with the exception of ABT-737, Bax or Bak are not necessary for cell death induction and most putative BCL2 inhibitors do not serve as $\mathrm{BH} 3$-mimetics or selective BCL2 inhibitors, which require Bak or Bax for cell death induction. ${ }^{31}$ Similar experiments with Bax/Bak DKO MEFs had been carried out earlier for chelerythrine, gossypol and several other putative BCL2 antagonists with comparable results. ${ }^{15,32,33}$ In one study obatoclax induced cell death was reported to be partially dependent on Bak expression. ${ }^{32}$ Therefore it is possible that in some cell types there may be a small contribution of Bax or Bak to the toxicity of these compounds, as we observed a slightly lower sensitivity particularly to gossypol, apogossypol and obatoclax of Bax/ Bak DKO compared with wt MEFs (Figure 1). Considering the higher potency of ABT-737 in CLL cells compared with the other inhibitors (Figure 3 ), it is possible that the development of analogs of gossypol, apogossypol and obatoclax with higher affinities for antiapoptotic BCL2 members will yield more potent and selective compounds, whose activity will also be more dependent on BAX/BAK. Furthermore, if BCL2 or $B C L-X_{L}$ exerts functions independently of BAX/BAK, such as binding of the voltage-dependent anion channel (VDAC) or inositol-3 phosphate receptor, then it is possible that these various inhibitors might still target antiapoptotic BCL2 proteins and inhibit some of these other functions. ${ }^{34,35}$ Therefore, we conclude that only $\mathrm{ABT}-737$ is a true $\mathrm{BH} 3-$ mimetic exerting its proapoptotic effect through BAX/BAK and most other BCL2 inhibitors must also target additional proteins outside the BCL2 family.

Furthermore, if these putative BCL2 antagonists bind specifically to antiapoptotic BCL2 proteins, they would be expected to induce cell death by releasing mitochondrial cytochrome $c$, formation of the Apaf-1 apoptosome and activation of caspases. Using cells deficient in caspase-9, the initiator caspase in the intrinsic apoptotic pathway, only ABT-737 but none of the other BCL2 inhibitors induced caspase-9-dependent cell death (Figure 2). These results imply that cell death induced by some of the other putative BCL2 antagonists may occur in a caspase-independent manner, in agreement with recent findings showing that cell death induced by gossypol and HA14-1 was not inhibited by the caspase inhibitor z-VAD.fmk. ${ }^{36-39}$ In contrast, ABT-737induced cell death was found earlier to require caspase

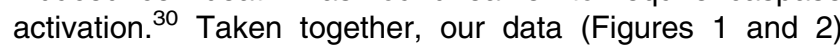
indicate that of the inhibitors tested only ABT-737 is a true inducer of apoptosis by activation of the intrinsic pathway, requiring both Bax/Bak and caspase-9. We suggest that despite the known ability of many of these putative BCL2 antagonists to bind to BCL2 proteins, most of these inhibitors are non-specific compounds and should not be used for mechanistic studies related to the function of BCL2 proteins. Dependent on their potency and toxicity, the agents investigated in this study might be valuable for cancer therapy irrespective of their mechanism of cell death induction. Although their potency was comparable in Jurkat T-cells, in CLL cells ABT-737 was 2-3 orders of magnitude more potent than any of the other compounds tested, indicating that CLL cells are exquisitely sensitive to the mechanism-based cell death induced by ABT-737 (Figures 2 and 3). In line with our results, sensitivity of CLL cells to obatoclax or gossypol was reported earlier at $1.7 \mu \mathrm{M}$ at $48 \mathrm{~h}^{40}$ and $\sim 30 \mu \mathrm{M}$ at $24 \mathrm{~h}$ of exposure, ${ }^{36}$ respectively, whereas sensitivity to $A B T-737$ was observed at nanomolar concentrations. ${ }^{11,13}$ Among the putative BCL2 inhibitors tested in this study, MEFs were most sensitive to obatoclax, which induced cell death at $100 \mathrm{nM}$ (Figure 1a and Supplementary Figure 1). Thus it is evident that the efficacy of BCL2 inhibitors, such as obatoclax, is markedly dependent on cell type, possibly reflecting the presence of additional targets outside the BCL2 family.

We have shown that the putative BCL2 inhibitors investigated in this study, with the exception of ABT-737, induce cell death independent of BCL2 proteins and activation of caspase-9. However, somewhat surprisingly, all these BCL2 inhibitors induced release of cytochrome $c$ and caspase cleavage (Figure 4), two commonly used biochemical indicators of apoptosis. Therefore, although most of these BCL2 inhibitors do not directly activate the intrinsic pathway of apoptosis, they nevertheless induce certain features of apoptosis, which could easily be mistaken as death by apoptosis. In this regard it was interesting to also consider the ultrastructural changes induced by these compounds (Figures 5 and 6). Obatoclax and chelerythrine induced rapid swelling of mitochondria with loss of cristae structure, which in the case of obatoclax occurred before induction of cell death. In line with these data, it has recently been shown that chelerythrine induces rapid release of cytochrome $c$ and mitochondrial swelling. ${ }^{33}$ With chelerythrine, obatoclax and gossypol, the mitochondrial damage was accompanied by phospholipid inclusions, which seemed to originate from the cristae membranes, raising the possibility that instead of specifically targeting BCL2 proteins, these compounds induce cell death by causing severe mitochondrial damage. In the present study, the appearance of these phospholipid inclusions was most evident in CLL cells exposed to chelerythrine for only $1 \mathrm{~h}$ (Figure $5 \mathrm{~d}$ ), when the concentric layers of phospholipid somewhat resembled the alterations in cristae observed in HeLa cells after loss of mitofilin. ${ }^{41}$ Mitofilin is an inner mitochondrial membrane protein, which has been proposed to control cristae morphology. Thus it is possible that some of the putative BCL2 antagonists, including chelerythrine, obatoclax and gossypol, perturb mitochondria by affecting proteins that control cristae morphology.

It is interesting that, all the putative BCL2 antagonists induce strikingly different ultrastructural changes in CLL cells (Figures 5 and 6), implying significant differences in the biochemical pathways by which they induce cell death. Agents like ABT-737 may cause relatively specific MOMP that results in the release of cytochrome $c$, activation of caspases and 
induction of biochemical and morphological changes characteristically associated with apoptosis. Other BCL2 antagonists, including obatoclax, chelerythrine and gossypol, may have additional targets and cause mitochondrial damage, which can be accompanied by release of cytochrome $c$ and ultrastructural changes associated with either apoptosis or necrosis. It is to be noted that, the extensive mitochondrial damage and loss of cristae membranes induced by some of these agents will also severely compromise the ability of the cells to generate ATP by oxidative phosphorylation so also impairing the ability of the cells to undergo apoptosis, which is an ATP-requiring process. ${ }^{42}$ Thus these different ultrastructures may be because of differing kinetics and extents of mitochondrial perturbation together with impaired ability to generate ATP so modifying the biochemical subroutines used by the dying cells. It is interesting that, our results highlight important limitations of solely using biochemical assays, including cytochrome $c$ release, caspase activation and PARP cleavage, to define apoptosis or the mechanism of cell death. As all the compounds induced both cytochrome $c$ release and caspase activation, in the absence of the ultrastructural studies, we would have been blissfully ignorant of the major cellular changes occurring including the phospholipid inclusions and the proliferation of the endoplasmic reticulum (Figures 5 and 6).

Besides their ability to induce cell death, it is also important to consider the potential in vivo toxicities of the BCL2 antagonists. The major toxicity due to ABT-263 is mechanism based and involves a dose-dependent induction of thrombocytopenia because of inhibition of BCL- $\mathrm{X}_{\mathrm{L}}$, which is critical for determining platelet survival. ${ }^{43}$ For all other putative BCL2 inhibitors, non-mechanism based toxicity might occur in vivo. Gossypol induces the generation of reactive oxygen species, which may be responsible for the induction of cell death. ${ }^{44}$ In vivo gossypol is known to cause male infertility, whereas obatoclax had been reported to induce both neurological symptoms in early clinical trials of patients with CLL as well as neuronal toxicity in mice, ${ }^{25,45}$ which might be because of targets outside the BCL2 family. In this regard a recent study showed that obatoclax exerted growth inhibitory effects at lower concentrations than induction of apoptosis and suggested that obatoclax targeted other proteins in addition to BCL2 family members. ${ }^{32}$

In summary, we suggest that many of the putative BCL2 antagonists, including some of those currently undergoing clinical trials, are mitochondrial toxins that induce cell death primarily by massive mitochondrial damage rather than specific MOMP and activation of the intrinsic pathway of apoptosis. Owing to their nonspecific effects, the use of these putative BCL2 antagonists for mechanistic studies related to the function of BCL2 proteins should be viewed with caution. Of the inhibitors we have examined, only ABT-737 acts as a $\mathrm{BH} 3-$ mimetic, inducing Bax/Bak-dependent activation of the intrinsic apoptotic pathway.

\section{Materials and Methods}

BCL2 inhibitors. Racemic gossypol (NSC\# 19048) and racemic apogossypol (NSC\# 736630) were kindly provided by Dr. VL Narayanan (Drug Synthesis and Chemistry Branch, Developmental Therapeutics Program, Division of Cancer Treatment and Diagnosis, National Cancer Institute, Bethesda, MD). EM20-25
(BCL2 inhibitor III) was from Calbiochem (Nottingham, UK) and chelerythrine from Sigma-Aldrich Ltd. (Dorset, UK). Obatoclax (GX15-070) and ABT-737 were kindly provided by Dr. Gordon Shore (GeminX Pharmaceuticals, Montreal, Canada) and Dr. Saul Rosenberg (Abbott Laboratories, IL, USA), respectively.

Cell culture. Wild type and Bax/Bak double knockout (DKO) MEFs were provided by Drs. A Strasser and D. Huang (The Walter and Eliza Hall, Institute of Medical Research, Melbourne, Australia), and cultured in DMEM medium supplemented with $5 \mathrm{mM} \mathrm{L-glutamine} \mathrm{and} 10 \%$ fetal calf serum (FCS) (all from Life Technologies, Inc, Paisley, UK). Jurkat T-cells deficient in caspase-9 were cultured in RPMI 1640 medium supplemented with $10 \%$ FCS and $5 \mathrm{mM} \mathrm{L}$ glutamine (Life Technologies, Inc.). ${ }^{26,27}$ Unlike some Jurkat cells, these caspase- 9 deficient cells express Bax. ${ }^{27}$ Peripheral blood samples from patients with CLL were obtained with patient consent and local ethical committee approval. Lymphocytes were purified and immediately cultured in RPMI 1640 medium supplemented with $10 \%$ FCS and $5 \mathrm{mM}$ L-glutamine (Life Technologies, Inc.) as described earlier. ${ }^{30}$

Determination of cell death and cytochrome $c$ release. Cell death was assessed either by phosphatidylserine (PS) externalization and staining with AnnexinV-FITC or AnnexinV-APC (Invitrogen, Paisley, UK) or by changes in scatter properties and FSC/SSC analysis. To assess release of cytochrome $c, 10 \times 10^{6}$ CLL cells were washed in cold PBS and resuspended in $100 \mu \mathrm{l}$ mitochondrial isolation buffer $\left(250 \mathrm{mM}\right.$ sucrose, $20 \mathrm{mM}$ Hepes, $\mathrm{pH} 7.4,5 \mathrm{mM} \mathrm{MgCl}_{2}$ and $10 \mathrm{mM}$ $\mathrm{KCl}$ ) containing $0.05 \%$ digitonin. Cells were left on ice for $10 \mathrm{~min}$ followed by centrifugation at $13000 \mathrm{rpm}$ for $3 \mathrm{~min}$. Subsequently, supernatant and pellets were analyzed by western blotting.

Western blotting. Western blot analysis was carried out using mouse anticytochrome $c$ Ab (BD Bioscience, San Diego, CA, USA), mouse anti- $\alpha$-tubulin Ab (Calbiochem), mouse anti-PARP Ab (Alexis Biochemicals, Nottingham, UK). Rabbit anti-caspase-9 and rabbit anti-caspase-3 Abs were kindly provided by Dr. Xiao-Ming Sun. ${ }^{46}$ Enhanced chemiluminescence was used for detection (GE Healthcare, Bucks, UK).

Electron microscopy. The ultrastructure of 4-11 samples of CLL cells from at least 2 different patients was examined for all the inhibitors shown in Figures 5 and 6 except for ABT-737 where cells from 21 different patients have been examined. Cells were fixed in $2 \%$ glutaraldehyde in $0.1 \mathrm{M}$ sodium cacodylate buffer $(\mathrm{pH} 7.4)$ at $4{ }^{\circ} \mathrm{C}$ overnight and postfixed with $1 \%$ osmium tetroxide $/ 1 \%$ potassium ferrocyanide for $1 \mathrm{~h}$ at room temperature. After fixation, cells were stained en bloc with $5 \%$ aqueous uranyl acetate overnight at room temperature, dehydrated, and embedded in Taab epoxy resin (Taab Laboratories Equipment Ltd., Aldermaston, UK). Electron micrographs of ultrathin sections were recorded using a QICAM 12-bit Mono Fast 1394 digital camera and QCapture-Pro software (MAG, Pleasanton, CA, USA) in a Zeiss 902A electron microscope.

Statistics. To compare cell death in wt or DKO MEFS or caspase-9 deficient and expressing Jurkat cells, an unpaired $t$-test was used. For $\mathrm{EC}_{50}$ values at $24 \mathrm{~h}$ of drug exposure, the specific apoptosis was calculated as follows: (drug-induced death-spontaneous death $) \times 100 /(100$-spontaneous death). For calculation of the $\mathrm{EC}_{50}$ values, a nonlinear equation was used. All statistics were carried out in GraphPad prism.

Acknowledgements. We thank Dr. Gordon Shore (GeminX Pharmaceuticals) and Dr. Saul Rosenberg (Abbott Laboratories) for providing us with obatoclax and ABT-737, respectively. We thank Dr. Andreas Strasser for providing Bax/Bak DKO MEFs. We thank Judy McWilliam and Tim Smith for preparation of material for EM. This work was supported by the Medical Research Council and grants from the Marie Curie Research Training Network 'ApopTrain' (KW and GMC) and the Leukaemia Research Fund.

1. Hanahan D, Weinberg RA. The hallmarks of cancer. Cell 2000; 100: 57-70.

2. Arends MJ, Wyllie AH. Apoptosis: mechanisms and roles in pathology. Int Rev Exp Pathol 1991; 32: 223-254.

3. Jaattela M. Multiple cell death pathways as regulators of tumour initiation and progression. Oncogene 2004; 23: 2746-2756.

4. Green DR, Kroemer G. The pathophysiology of mitochondrial cell death. Science 2004; 305: 626-629. 
5. Cohen GM. Caspases: the executioners of apoptosis Biochem J 1997; 326: 1-16.

6. Wei MC, Zong WX, Cheng EH, Lindsten T, Panoutsakopoulou V, Ross AJ et al Proapoptotic $\mathrm{BAX}$ and $\mathrm{BAK}$ : a requisite gateway to mitochondrial dysfunction and death Science 2001; 292: 727-730.

7. Adams JM, Cory S. The Bcl-2 apoptotic switch in cancer development and therapy. Oncogene 2007; 26: 1324-1337.

8. Amundson SA, Myers TG, Scudiero D, Kitada S, Reed JC, Fornace Jr AJ. An informatics approach identifying markers of chemosensitivity in human cancer cell lines. Cancer Res 2000; 60: 6101-6110.

9. Vogler M, Dinsdale D, Dyer MJ, Cohen GM. Bcl-2 inhibitors: small molecules with a big impact on cancer therapy. Cell Death Differ 2009; 16: 360-367.

10. Zeitlin BD, Zeitlin IJ, Nor JE. Expanding circle of inhibition: small-molecule inhibitors of Bcl2 as anticancer cell and antiangiogenic agents. J Clin Oncol 2008; 26: 4180-4188.

11. Oltersdorf T, Elmore SW, Shoemaker AR, Armstrong RC, Augeri DJ, Belli BA et al. An inhibitor of Bcl-2 family proteins induces regression of solid tumours. Nature 2005; 435 677-681.

12. Bruncko M, Oost TK, Belli BA, Ding H, Joseph MK, Kunzer A et al. Studies leading to potent, dual inhibitors of Bcl-2 and Bcl-xL. J Med Chem 2007; 50: 641-662.

13. Del Gaizo Moore V, Brown JR, Certo M, Love TM, Novina CD, Letai A. Chronic lymphocytic leukemia requires $B C L 2$ to sequester prodeath $B I M$, explaining sensitivity to $B C L 2$ antagonist ABT-737. J Clin Invest 2007; 117: 112-121.

14. Tahir SK, Yang X, Anderson MG, Morgan-Lappe SE, Sarthy AV, Chen J et al. Influence of Bcl-2 family members on the cellular response of small-cell lung cancer cell lines to ABT737. Cancer Res 2007; 67: 1176-1183.

15. van Delft MF, Wei AH, Mason KD, Vandenberg CJ, Chen L, Czabotar PE et al. The BH3 mimetic ABT-737 targets selective Bcl-2 proteins and efficiently induces apoptosis via Bak/ Bax if Mcl-1 is neutralized. Cancer Cell 2006; 10: 389-399.

16. Nguyen M, Marcellus RC, Roulston A, Watson M, Serfass L, Murthy Madiraju SR et al. Small molecule obatoclax (GX15-070) antagonizes MCL-1 and overcomes MCL-1. mediated resistance to apoptosis. Proc Natl Acad Sci USA 2007; 104: 19512-19517.

17. Kitada S, Leone M, Sareth S, Zhai D, Reed JC, Pellecchia M. Discovery, characterization, and structure-activity relationships studies of proapoptotic polyphenols targeting B-cell lymphocyte/leukemia-2 proteins. J Med Chem 2003; 46: 4259-4264.

18. Becattini B, Kitada S, Leone M, Monosov E, Chandler S, Zhai D et al. Rational design and real time, in-cell detection of the proapoptotic activity of a novel compound targeting $\mathrm{Bcl}$ X(L). Chem Biol 2004; 11: 389-395.

19. Chan SL, Lee MC, Tan KO, Yang LK, Lee AS, Flotow $\mathrm{H}$ et al. Identification of chelerythrine as an inhibitor of BcIXL function. J Biol Chem 2003; 278: 20453-20456.

20. Milanesi E, Costantini P, Gambalunga A, Colonna R, Petronilli V, Cabrelle A et al. The mitochondrial effects of small organic ligands of $B C L-2$ : sensitization of $B C L-2$ overexpressing cells to apoptosis by a pyrimidine-2,4,6-trione derivative. J Biol Chem 2006; 281: 10066-10072.

21. Kitada S, Kress CL, Krajewska M, Jia L, Pellecchia M, Reed JC. Bcl-2 antagonis apogossypol (NSC736630) displays single-agent activity in Bcl-2-transgenic mice and has superior efficacy with less toxicity compared with gossypol (NSC19048). Blood 2008; 111: 3211-3219.

22. Herbert JM, Augereau JM, Gleye J, Maffrand JP. Chelerythrine is a poten and specific inhibitor of protein kinase C. Biochem Biophys Res Commun 1990; 172: 993-999.

23. Wilson WH, Tulipe A, Levine AM, Dunleavy K, Krivoshik AP, Hagey AE et al. A phase 1/2a study evaluating the safety, pharmacokinetics and efficacy of ABT-263 in subjects with refractory or relapsed lymphoid malignancies. Blood 2007; 110 Abstract 1371.

24. Mohammad R, Giri A, Goustin AS. Small-molecule inhibitors of Bcl-2 family proteins as therapeutic agents in cancer. Recent Patents Anticancer Drug Discov 2008; 3: 20-30.

25. O'Brien SM, Claxton DF, Crump M, Faderl S, Kipps T, Keating MJ et al. Phase I study of obatoclax mesylate (GX15-070), a small molecule pan-Bcl-2 family antagonist, in patients with advanced chronic lymphocytic leukemia. Blood 2009; 113: 299-305.
26. Samraj AK, Keil E, Ueffing N, Schulze-Osthoff K, Schmitz I. Loss of caspase-9 provides genetic evidence for the type I/II concept of CD95-mediated apoptosis. J Biol Chem 2006; 281: 29652-29659.

27. Samraj AK, Sohn D, Schulze-Osthoff K, Schmitz I. Loss of caspase-9 reveals its essential role for caspase-2 activation and mitochondrial membrane depolarization. Mol Biol Cell 2007; 18: 84-93.

28. Inoue S, Riley J, Gant TW, Dyer MJ, Cohen GM. Apoptosis induced by histone deacetylase inhibitors in leukemic cells is mediated by Bim and Noxa. Leukemia 2007; 21: 1773-1782.

29. Brown DG, Sun XM, Cohen GM. Dexamethasone-induced apoptosis involves cleavage of DNA to large fragments prior to internucleosomal fragmentation. J Biol Chem 1993; 268 : 3037-3039.

30. Vogler M, Dinsdale D, Sun XM, Young KW, Butterworth M, Nicotera $P$ et al. A novel paradigm for rapid $A B T-737$-induced apoptosis involving outer mitochondrial membrane rupture in primary leukemia and lymphoma cells. Cell Death Differ 2008; 15: 820-830.

31. Willis SN, Adams JM. Life in the balance: how BH3-only proteins induce apoptosis. Curr Opin Cell Biol 2005; 17: 617-625

32. Konopleva M, Watt J, Contractor R, Tsao T, Harris D, Estrov Z et al. Mechanisms of antileukemic activity of the novel Bcl-2 homology domain-3 mimetic GX15-070 (obatoclax). Cancer Res 2008; 68: 3413-3420.

33. Wan KF, Chan SL, Sukumaran SK, Lee MC, Yu VC. Chelerythrine induces apoptosis through a Bax/Bak-independent mitochondrial mechanism. J Biol Chem 2008; 283: 8423-8433.

34. Tsujimoto Y. Bcl-2 family of proteins: life-or-death switch in mitochondria. Biosci Rep 2002; 22: $47-58$

35. White C, Li C, Yang J, Petrenko NB, Madesh M, Thompson CB et al. The endoplasmic reticulum gateway to apoptosis by $\mathrm{Bcl}-\mathrm{X}(\mathrm{L})$ modulation of the InsP3R. Nat Cell Biol 2005; 7: 1021-1028.

36. Balakrishnan K, Wierda WG, Keating MJ, Gandhi V. Gossypol, a BH3 mimetic, induces apoptosis in chronic lymphocytic leukemia cells. Blood 2008; 112: 1971-1980.

37. Zhang M, Liu H, Tian Z, Griffith BN, Ji M, Li QQ. Gossypol induces apoptosis in human PC3 prostate cancer cells by modulating caspase-dependent and caspase-independent cell death pathways. Life Sci 2007; 80: 767-774.

38. Oliver L, Mahe B, Gree R, Vallette FM, Juin P. HA14-1, a small molecule inhibitor of Bcl-2, bypasses chemoresistance in leukaemia cells. Leuk Res 2007; 31: 859-863.

39. Chen J, Freeman A, Liu J, Dai Q, Lee RM. The apoptotic effect of HA14-1, a Bcl-2interacting small molecular compound, requires Bax translocation and is enhanced by PK11195. Mol Cancer Ther 2002; 1: 961-967.

40. Campas C, Cosialls AM, Barragan M, Iglesias-Serret D, Santidrian AF, Coll-Mulet $L$ et al. $\mathrm{Bcl}-2$ inhibitors induce apoptosis in chronic lymphocytic leukemia cells. Exp Hematol 2006; 34: $1663-1669$

41. John GB, Shang Y, Li L, Renken C, Mannella CA, Selker JM et al. The mitochondrial inner membrane protein mitofilin controls cristae morphology. Mol Biol Cell 2005; 16: 1543-1554.

42. Leist M, Single B, Castoldi AF, Kuhnle S, Nicotera P. Intracellular adenosine triphosphate (ATP) concentration: a switch in the decision between apoptosis and necrosis. J Exp Med 1997; 185: 1481-1486.

43. Mason KD, Carpinelli MR, Fletcher Jl, Collinge JE, Hilton AA, Ellis S et al. Programmed anuclear cell death delimits platelet life span. Cell 2007; 128: 1173-1186.

44. Ko CH, Shen SC, Yang LY, Lin CW, Chen YC. Gossypol reduction of tumor growth through ROS-dependent mitochondria pathway in human colorectal carcinoma cells. Int J Cancer 2007; 121: 1670-1679.

45. Trudel S, Li ZH, Rauw J, Tiedemann RE, Wen XY, Stewart AK. Preclinical studies of the pan-Bcl inhibitor obatoclax (GX015-070) in multiple myeloma. Blood 2007; 109: $5430-5438$.

46. Sun XM, MacFarlane M, Zhuang J, Wolf BB, Green DR, Cohen GM. Distinct caspase cascades are initiated in receptor-mediated and chemical-induced apoptosis. $J$ Biol Chem 1999; 274: 5053-5060.

\section{Supplementary Information accompanies the paper on Cell Death and Differentiation website (http://www.nature.com/cdd)}

